\title{
Editorial: Zooplankton and Nekton: Gatekeepers of the Biological Pump
}

\begin{abstract}
Rainer Kiko ${ }^{*}$, Daniele Bianchi ${ }^{2}$, Christian Grenz ${ }^{3}$, Helena Hauss ${ }^{4}$, Morten Iversen ${ }^{5,6}$, Sanjeev Kumar ${ }^{7}$, Amy Maas ${ }^{8}$ and Carol Robinson ${ }^{9}$

${ }^{1}$ Sorbonne Université, Laboratoire d'Océanologie de Villefranche-sur-Mer, Villefranche-sur-Mer, France, ${ }^{2}$ Atmospheric \& Oceanic Sciences, University of California, Los Angeles, Los Angeles, CA, United States, ${ }^{3}$ Aix Marseille Université, Université Toulon, CNRS/INSU, IRD, Mediterranean Institute of Oceanography MIO UM 110, Marseille, France, ${ }^{4}$ GEOMAR Helmholtz Centre for Ocean Research Kiel, Kiel, Germany, ${ }^{5}$ Polar Biological Oceanography, Alfred Wegener Institut, Bremerhaven, Germany, ${ }^{6}$ MARUM and University of Bremen, Bremen, Germany, ${ }^{7}$ Physical Research Laboratory, Ahmedabad, India, ${ }^{8}$ Bermuda Institute of Ocean Sciences, St. Georges, Bermuda, ${ }^{9}$ School of Environmental Sciences, University of East Anglia, Norwich, United Kingdom
\end{abstract}

Keywords: zooplankton, nekton, biological pump, editorial, global ocean, zooplankton-particle interactions, export flux

\section{Editorial on the Research Topic}

\section{Zooplankton and Nekton: Gatekeepers of the Biological Pump}

Zooplankton and nekton organisms create and destroy particles in manifold ways. They feed on the diverse components of the plankton community and on detrital matter. They disaggregate these components, but also repackage them into fecal pellets. Zooplankton and nekton thereby contributes to the attenuation, but also to the export of vertically settling particles. Many zooplankton and nekton organisms also ascend to the surface layer of the ocean at dusk to feed during the dark hours, and return to midwater at the break of dawn. This diurnal vertical migration (DVM) shuttles organic matter from the surface ocean to deeper layers, where it is metabolized and excreted. This active flux (as opposed to the passive flux of sinking particles) can contribute substantially to the biological pump, the downward export of carbon and nutrients into the oceans interior. Due to their multiple roles in oceanic particle dynamics, zooplankton and nekton organisms can actually be considered the gatekeepers of the biological pump.

Several articles in this Research Topic deal with the contribution of zooplankton and nekton-mediated active flux to the total export of organic matter. Using biomass and enzyme transport system (ETS) assessments of respiratory flux for both mesozooplankton and micronekton communities, Hernández-León et al. estimated the total active transport of carbon (respiration, excretion, mortality, and egestion) along a transect in the Atlantic from the Canary Islands to Brazil. They found that active flux by these communities ranged from 25 to $80 \%$ of the total particulate organic carbon export at $150 \mathrm{~m}$ depth and that the importance of active flux increased with increasing surface productivity. Kwong et al. compared biomass, diel vertical migration, and active flux of mesozooplankton and micronekton across a range of mesoscale eddy structures along the east-coast of Australia during winter and spring. They found that although all eddy regimes had similar integrated biomass of mesozooplakton and micronekton, the organisms in the individual eddies had different migratory behavior, which resulted in contrasting importance of active flux. Kiko et al. assessed the impact of mesozooplankton DVM on elemental cycling at three stations in the Eastern Tropical North Atlantic. They found that approximately 31 to $41 \%$ of the total nitrogen loss from the upper $200 \mathrm{~m}$ of the water column was attributable to DVM mediated fluxes. They also suggest that gut flux - the flux created by migrators when they evacuate their gut at DVM-depth-and migrator mortality at DVM-depth contribute to an Intermediate Particle Maximum. In their study conducted in the Peruvian upwelling system (which features a severe midwater oxygen minimum zone), Kiko and Hauss concluded that the metabolic suppression 
of zooplankton metabolic activity at low oxygen needs to be considered when calculating active fluxes. This metabolic suppression results in a substantial reduction of both respiration and ammonium excretion within the OMZ core. Kelly et al. employed a linear inverse ecosystem model to explore carbon fluxes between the epipelagic and the mesopelagic zones in the California Current Ecosystem. The model was constrained by measurements from quasi-Lagrangian experiments spanning a wide range of conditions from upwelling to oligotrophic regions. They consistently found that active transport by mesozooplankton was important in supporting the mesopelagic carbon demand, and also highlighted the central importance of mesozooplankton in marine food webs.

During an austral summer cruise in the Southern Ocean, Liszka et al. studied the peculiarities of fecal pellet transport in the upper mesopelagic layer. They confirmed that zooplankton contributed to the carbon supply to deeper waters, but also ascertained their role in the breakdown and reworking of the fecal pellets as they sink. Stukel et al. used MOCNESS and UVP5 data combined with allometric equations to characterize the impact of different suspension and flux feeders on particle flux attenuation (assessed using drifting sediment traps and thorium isotope measurements) in the California upwelling ecosystem. They found that suspension feeders have a large impact on small, slow sinking particles, whereas the two investigated flux feeders (the pteropod Limacina helicina and phaeodarian Aulophaeridae) attenuated the flux of large, presumably fast settling particles, by about 10 to $20 \%$ within the upper $100 \mathrm{~m}$ of the water column. Toullec et al. found that micro-turbulence created by the swimming activity of small copepods encouraged aggregate formation of suspended diatoms, while the stronger turbulence created by large, cruise feeding copepods prevented aggregation and caused large aggregates to fragment. This work may cast new light on the observations of phytoplankton aggregates being an important vector for carbon export at low latitudes, whereas zooplankton fecal pellets seem to be an important contributor to carbon flux at high latitudes.

Finally, Robison et al. used MBARI's extensive in situ ROV observations of organismal behaviors and abundances to calculate the threat potential to the migratory community from encounters with mid-water predators. Although these estimates do not yet provide a quantitative prediction of the mortality experienced by migrators, they help us to better comprehend this very poorly-sampled, but likely substantial, component of active flux.

A general theme in all contributions in this Research Topic is the need for size- and/or weight-specific parameterizations to estimate biomass, gut flux, mortality, predation potential, excretion, respiration or flux-interception potential. Efforts should be strengthened to collect, synthesize and compare these parameters, but further efforts are also needed to harmonize and share zooplankton and nekton distribution data across studies. Such efforts will enable modeling studies and model assessments, which could yield a more comprehensive understanding of the role of zooplankton and nekton as essential components of the biological pump.

\section{AUTHOR CONTRIBUTIONS}

All authors listed have made a substantial, direct and intellectual contribution to the work, and approved it for publication.

\section{FUNDING}

RK was supported by a Make Our Planet Great Again grant of the French National Research Agency within the Programme d'Investissements d'Avenir: reference ANR-19-MPGA-0012. MI was supported by the HGF Young Investigator Group SeaPump Seasonal and Regional Food Web Interactions with the Biological Pump: VH-NG-1000. HH received support from iAtlantic (EU H2020 grant agreement no. 818123) and from project CUSCO of the German Federal Ministry of Education and Research (BMBF). CR was supported by funds from The Leverhulme Trust (Grant RPG-2017-089) and the Natural Environment Research Council (NERC) of the United Kingdom (Grant NE/R000956/1). DB acknowledges support by the U.S. National Science Foundation under Grant 1635632. Funding for AM was provided by the NASA EXPORTS project (grant 80NSSC17K0654).

\section{ACKNOWLEDGMENTS}

We would like to thank the Frontiers team for their support and the following reviewers for their contribution to this Research Topic: Bingzhang Chen, John P. Dunne, Santiago Hernández-León, Kusum Komal Karati, Qian Li, Daria Martynova, Natalia Osma, Suzanne J. Painting, Jyothibabu Retnamma, HS, Rudi Schuech, Michael R. Stukel, Jun Sun, Geraint A. Tarling, and Xiaodong Wang.

Conflict of Interest: The authors declare that the research was conducted in the absence of any commercial or financial relationships that could be construed as a potential conflict of interest.

Copyright (c) 2020 Kiko, Bianchi, Grenz, Hauss, Iversen, Kumar, Maas and Robinson. This is an open-access article distributed under the terms of the Creative Commons Attribution License (CC BY). The use, distribution or reproduction in other forums is permitted, provided the original author(s) and the copyright owner(s) are credited and that the original publication in this journal is cited, in accordance with accepted academic practice. No use, distribution or reproduction is permitted which does not comply with these terms. 